\title{
Beat Number
}

National Cancer Institute

\section{Source}

National Cancer Institute. Beat Number. NCI Thesaurus. Code C69283.

The number of a specific beat. 